\title{
Unscheduled return visits within 72 hours to an assessment unit
}

\author{
M K Lal, M S Kibirige
}

\begin{abstract}
Unscheduled return visits were looked at to determine the quality of care and safety of patients in a paediatric assessment unit. The reasons for unscheduled return visits were also investigated. Two per cent of patients discharged from the unit returned, the main reason being parental perception of illness. There were only two patients re-referred by their family doctor. These findings have implications for clinical care and education.

(Arch Dis Child 1999;80:455-458)
\end{abstract}

Keywords: assessment unit; unscheduled return visits

During the past decade there has been a substantial increase in the number of children referred to hospitals for admission. ${ }^{12}$ This trend continues despite improving social and economic conditions for most children, improved health, falling infant and child mortality, reduced length of stay in hospital, better parental health education, improved training in paediatrics for general practitioners, and increase in general practitioner consultation rates and availability.

Short stay facilities such as admission units, assessment units, or day care units exist at many hospitals within the UK to deal with acute referrals. These facilities have been found to help in patient management. ${ }^{345}$ They rationalise admissions by ensuring prompt treatment and transfer to the ward when admission is required, or allow children to return home the same day, thus reducing pressure on parents. Against this background, however, there are concerns that some children might be discharged too early, or inappropriately, thus compromising their care.

In an attempt to address this issue objectively, the paedia tric appropriateness evaluation protocol has been developed, ${ }^{6}$ but proved to have limited validity for evaluating British paediatric practice and so is not recommended. ${ }^{7}$ Other tools are being evaluated.

Our hospital provides a district paediatric service for a population of approximately 85000 children. The district covers many areas with a high social deprivation index as highlighted by Phillimore et al. ${ }^{8}$ Our hospital has also been experiencing a rising number of patient referrals. There was a $15 \%$ increase in the number of referrals in 1991-92 compared with 1990-91. This trend has continued at $22 \%$ in $1994-95,7.5 \%$ in 1995-96, and $41 \%$ in $1996-97$, compared with the previous year.
An assessment unit was set up to deal with the problem of increasing referrals. The philosophy was to admit only those children whose needs could only be met in a hospital. It also provides an area of direct access to the referring teams so that each case being referred can be discussed. The referring teams include general practitioners and an accident and emergency department located elsewhere. There are arrangements with the ambulance service to ensure that all emergency non-trauma patients are brought directly to the unit.

It also provides a place where patients can be investigated or observed without resorting to admission. The unit's staff has no other inpatient duties.

\section{Unit staff and facilities}

The unit is a designated area, adjacent to the children's wards. It consists of seven bed spaces and is equipped with a resuscitation room, a treatment area, and two administrative offices. There are seven full time equivalent nursing staff assisted by two auxiliary nurses. We have two extra community nurses and specialist nurses for diabetes, cystic fibrosis, asthma, epilepsy, and critical care. The unit is consultant led with one senior house officer and a registrar available during the opening hours between 0900 and 2100 hours on weekdays and 1000 and 1700 hours at weekends. The unit was first opened in November 1994. At that time we only operated on weekdays.

\section{Operational policy of the unit}

Clinical assessment entails obtaining details of the presenting complaints, in addition to social and past medical history, as well as contact telephone number and availability of own transport. The decision to admit an individual patient is based on clinical need, whereby the child's management requirements can only be met within a hospital environment. Social circumstances sometimes influence the decision and the parents have an opportunity to say if they feel comfortable with looking after their child at home.

Appropriate management is outlined to the parents and arrangements are made either to see the family doctor, a community nurse, a specialist nurse where appropriate, or for a formal discharge. Health education and information leaflets are provided as necessary. When a child is sent home, parents are advised to contact the unit in the event of deterioration or lack of improvement. Discharge from the unit is effected by an experienced member of staff of registrar grade or above. 


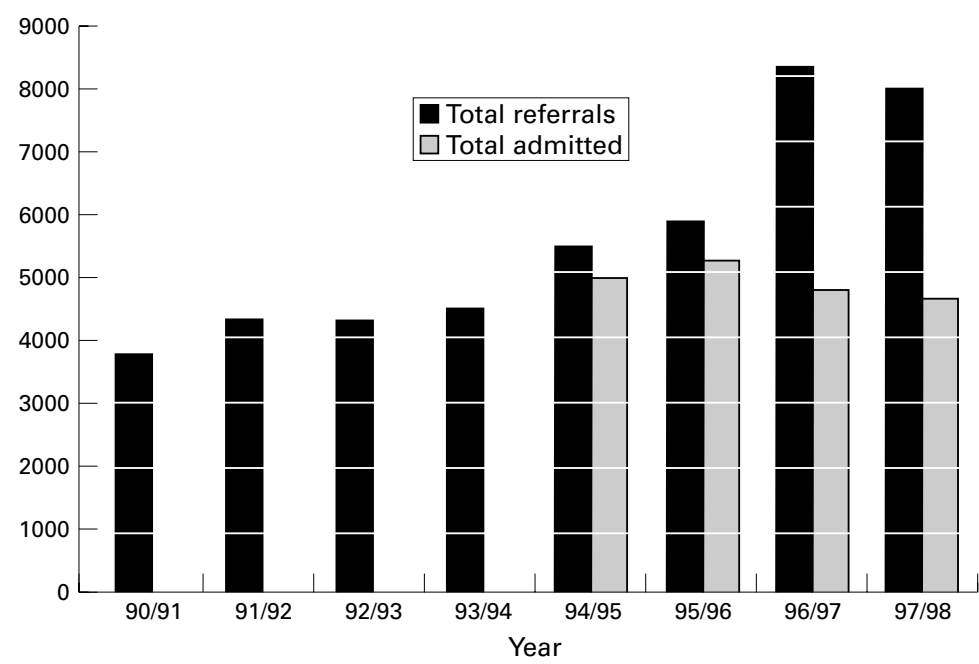

Figure 1 Trends in referral and admission 1991-98. The time intervals represent fiscal years (April to March). The study period was between Fanuary 1995 and fune 1997.

To determine the quality of service provided, we decided to investigate unscheduled return visits as an indicator of outcome after discharge from the assessment unit.

\section{Methods and analysis}

Our study was conducted over a 30 month period from January 1995 to June 1997. All demographic details, in addition to the reasons for and outcome of unscheduled return visits, were collected prospectively and stored on a computerised database.

The unscheduled return visits were identified using three main sources of information. These were a hand written register based in the unit, the hospital computerised patient administration system used with the medical records for hospital numbers, and the interdistrict hospital information register. The last was established to monitor how many children have to travel to other districts because of a lack of beds. This helped us to determine if any of the discharged patients had transferred their care to a neighbouring hospital. Information is fed back on a monthly basis. The information gathered on unscheduled return visits has been analysed and compared with historical data.

\section{Results}

Figure 1 shows the trend of referrals and admissions before and after the unit opened. Before the opening of the unit, referral equated

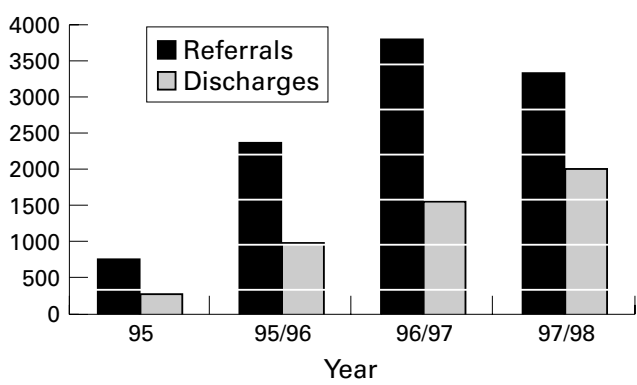

Figure 2 Acute referrals and discharges (assessment unit) 1995-98. The time intervals represent fiscal years (April to March). The study period was between fanuary 1995 and fune 1997.

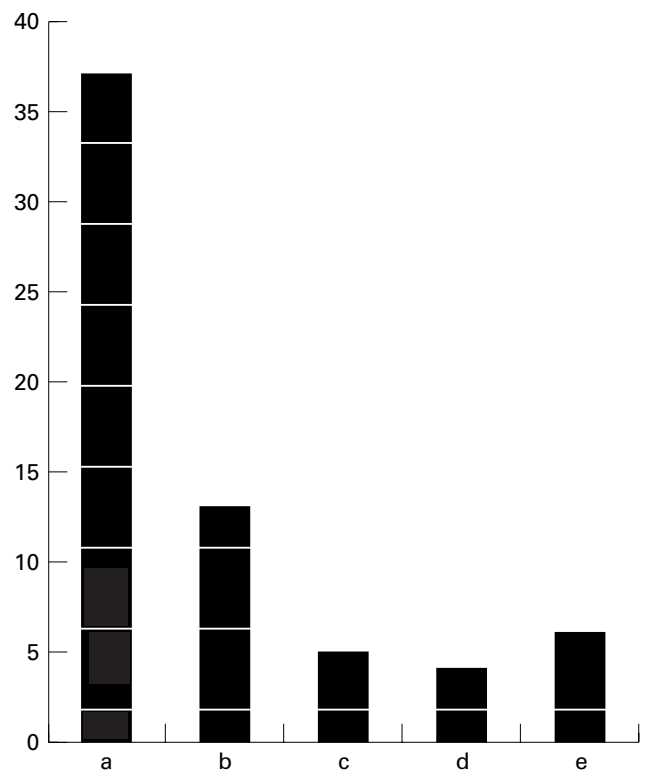

Figure 3 Reasons for unscheduled return visits. $a$, persistence of symptoms; $b$, failure to respond to treatment; $c$, change in clinical presentation; $d$, unrelated medical diagnosis; e, social and miscellaneous.

to admission to the children's wards where assessment and observation were carried out. Despite a sharp increase in acute referrals, we have managed to slow down the admission rate.

During the study period, 7328 patients were referred for admission. Of these, 3131 (43\%) were allowed home after initial assessment. Figure 2 shows the trend for discharges from the assessment unit in relation to the increase in referral rates.

There were 106 children requested to return for review. All these scheduled returns were seen as arranged and formally discharged.

We identified $65(2 \%)$ unscheduled return visits during this period. The interdistrict hospital register did not reveal any children discharged from the unit who went to either of the two neighbouring hospitals. The reasons for their return are outlined in fig 3. The diagnostic spectrum in these patients is outlined in table 1 , and is no different from that seen in acute referrals to our unit in general.

Table 1 Diagnosis of acute referrals and unscheduled return visits (URVs)

\begin{tabular}{llll}
\hline & $\begin{array}{l}\text { Acute referrals } \\
(n=7328)\end{array}$ & & $\begin{array}{l}\text { URVs } \\
(n=65)\end{array}$ \\
\cline { 2 - 2 } Diagnosis & Number (\%) & & Number (\%) \\
\hline Respiratory & $1634(22.3)$ & & $22(33.8)$ \\
CNS/epilepsy & $740(10.1)$ & & $4(6.1)$ \\
Gastrointestinal & $740(10.1)$ & & $16(24.6)$ \\
Severe multisystem infection & $615(8.4)$ & & $1(1.5)$ \\
Congenital & $571(7.8)$ & & 0 \\
Haematology/oncology & $447(6.1)$ & & $2(3)$ \\
Endocrine & $300(4.1)$ & & $1(1.5)$ \\
Accidental poisoning & $256(3.5)$ & & $1(1.5)$ \\
Dermatological & $227(3.1)$ & & $5(7.6)$ \\
UTI/genitourinary & $219(3.0)$ & & $10(15.4)$ \\
Cardiovascular & $175(2.4)$ & & 0 \\
Musculoskeletal & $110(1.5)$ & & 0 \\
Psychosocial & $132(1.8)$ & & 0 \\
Surgical & $1162(15.8)$ & $3(4.6)$ \\
\hline
\end{tabular}

CNS, central nervous system; UTI, urinary tract infection. 
Table 2 Outcome of children admitted to ward requiring only observation ( $<24$ hours)

\begin{tabular}{ll}
\hline Age & Diagnosis \\
\hline 9 months & Gastroenteritis \\
7 years & Herpes stomatitis \\
4 years & Constipation \\
$91 / 2$ years & Abdominal pain \\
22 months & Accidental poisoning \\
22 months & Gastroenteritis \\
7 years & Henoch-Schönlein purpura \\
6 weeks & Gastroenteritis \\
21 months & Gastroenteritis \\
2 months & Hypothermia, bilateral inguinal hernia \\
2 months & Vomiting \\
2 months & Cystic hygroma \\
\hline
\end{tabular}

Of the 65 unscheduled return visits, 20 children were subsequently admitted to the ward. Tables 2 and 3 summarise the details of their problems. Twelve of these cases required observation only, and only four remained on the ward for more than 24 hours.

\section{Discussion}

Unscheduled return visits constituted only $2 \%$ of the discharges from the assessment unit. In spite of increased referrals, the assessment unit has produced a slow down in the percentage of patients receiving inpatient care. Our results indicate that the process is safe if well run and supervised. There were no deaths and no patient required intensive care as a result of decisions made in the unit. We have evidence that no patient opted to go to neighbouring hospitals because of decisions made in the assessment unit.

Parents' perception of illness was the main reason for returning to the unit. This highlights that a full assessment must ensure that parents' concerns are listened to and that they are involved in decision making about the care of their children.

This particular aspect is evident in some of the 20 children who made unscheduled return visits, and who spent a night in hospital for observation. This period of observation in the ward reassured parents and gave them confidence to care for their children at home. In 16 of the 20 unscheduled return visits, the parents' perception had been that the child could not have been managed at home.

Our experience regarding the care of children in this setting is similar to that observed elsewhere in the country. ${ }^{34}$ There has been an increase in the number of patients sent home directly from the unit. This has in turn led to a reduction in the percentage of children receiving inpatient care.

There have been concerns about self referrals. These are of two categories, patients

Table 3 Outcome of children admitted to ward requiring intervention

\begin{tabular}{lll}
\hline Age & Diagnosis & Intervention \\
\hline 10 years & Torsion of cyst of Morgagni ${ }^{\star}$ & Cyst removal \\
9 months & Viral croup & Nebulised budesonide \\
10 years & Abdominal pain & Normal abdominal ultrasonogram \\
4 months & Bronchiolitis & Nasogastric tube feeding \\
$91 / 2$ years & Headache & CT scan head normal \\
3 years & Craniopharyngioma & MRI scan brought forward \\
15 months & Chicken pox, pneumonia & Antibiotic \\
4 years & Tonsillitis & Antibiotic \\
\hline
\end{tabular}

$\star$ Ward stay $<24$ hours.

CT, computed tomography; MRI, magnetic resonance imaging. whose parents bypass the primary care service and the unscheduled return visits. We discourage the former but it is important to allow the latter access to the service if parents remain concerned. Our data show that two of the 18 self referrals had major problems requiring intervention. The provision of access to those who have been discharged is an extra safety measure.

Supportive services, community nurses, and specialist nurses were put in place to ensure patient safety and this has given confidence to parents and general practitioners. It also ensured that the ambulatory service continues to thrive.

A 72 hour period is used as our cut off point for returning to the unit unless it is a scheduled appointment. This was based on the assumption that those patients who return during this period are more likely to have symptoms related to the original complaint. It provides safety for those patients who may have been seen during the prodrome phase of an illness. It deals with most patients who deteriorate or those who do not respond to the prescribed management.

Although we do not have data on the number of children who went back to see their general practitioners, we are confident that the offered access ensures return to the unit rather than to other hospitals.

Our findings have implications for clinical service provision. Consultation in the unit has to focus on the issues that most concern parents or carers. Understanding the problem and methods used to manage it are crucial to the success of ambulatory care. Sufficient time must be provided to explain in detail, ${ }^{9}$ and clearly written information should be provided for parents to refer to at home.

The availability of an assessment unit could encourage many health workers in the primary care sector to seek a second opinion for conditions they otherwise might have managed on their own. It is possible that parents may demand a second opinion. We are monitoring this to see whether it might partly explain the continued escalation of referrals, although a similar trend has been observed in other studies. $^{4}$

The workload created has to be handled, so staffing issues have to be evaluated regularly. Education for parents and communication with other health workers must be evaluated regularly to ensure continuity of care for children. Management guidelines are shared and parents' participation is ensured. ${ }^{9}$

In summary, the assessment unit has been effective in reducing patient admissions, with few unscheduled return visits and no major problems as a result of decisions made to manage children at home.

1 Durojaiye LIA, Hutchinson T, Madeley RJ. Improved primary care does not prevent the admission of children to hospital. Public Health 1989;103:181-8.

2 Hill AM. Trends in paediatric medical admissions. BMF 1989;298:1479-83.

3 Browne GJ, Penna A. Short stay facilities: the future of efficient paediatric emergency services. Arch Dis Child 1996;74:309-13. 
4 Meates M. Ambulatory paediatrics-making a difference. Arch Dis Child 1997;76:468-76.

5 Beverley DW, Ball RJ, Smith the experience of implementing a children's day assessmen unit in a district general hospital. Arch Dis Child 1997;77:287-93.

6 Kreger BE, Restuccia JD. Assessing the need to hospitalize children: paediatric appropriateness evaluation protocol Pediatrics 1989;84:242-7.
7 Werneke U, Smith H, Smith IJ, Taylor J, MacFaul R. Validation of the paediatric appropriateness evaluation protocol in British practice. Arch Dis Child 1997;77: 294-8.

8 Phillimore P, Beattie A, Townsend P. Widening inequality of health in northern England. BMF 1994;308:1125-8.

9 Pachter LM. Parents' participation and perspectives regarding clinical judgment and clinical guideline development. Curr Opin Paediatr 1998;10:476-9.

\section{FETAL AND NEONATAL EDITION}

\section{May issue}

The following articles-being published in the May 1999 issue of the Fetal and Neonatal edition of the Archives of Disease in Childhood - may be of general interest to paediatricians.

Randomised controlled trial of cisapride in preterm infants

$R \mathcal{F} M c$ Clure, $\mathcal{F} H$ Kristensen, $A$ Grauaug

Body composition in preterm infants during infancy

$R \mathcal{F}$ Cooke, K McCormick, I f Griffin, D $\mathcal{F}$ Rawlings, K Faulkner, $\mathcal{F} C \mathrm{~K}$ Wells, $\mathcal{F} S$ Smith, $S \mathcal{F}$ Robinson

Permanent neonatal diabetes mellitus: clinical presentation and epidemiology in Oman

Bhasker Bappal, Palany Raghupathy, Vasantha de Silva, Saleh Mohamed Al Khusaiby

Fetal and infant death in mono- and dizygotic twins in England and Wales 1982-91

$C R$ West, Y Adi, P O D Pharoah

Relation between size of delivery unit and neonatal death in low risk deliveries: population based study

Dag Moster, Rolv Terje Lie, Trond Markestad

UK neonatal intensive care services in 1996

fanet Tucker, William Tarnow-Mordi, Craig Gould, Gareth Parry, Neil Marlow, on behalf of the UK Neonatal Staffing Study Collaborative Group

Ultrasound study of heel to calcaneum depth in neonates

Anoo fain, Nicholas Rutter 